Mini Review

\title{
Recent Research and Advances of Gradient Graphene and 3D Collectors for Lithium Metal Anode
}

Yuan Ma*, Yingying Jia, Yi Zhang, Fangzheng Qin, Mingjie He, Xiaohang Ma, Lingyun Liu, Yaodong Wu, Ying Meng, Zhenfa Zi ${ }^{*}$

Key Laboratory for Photoelectric Detection Science and Technology of Education Department of Anhui Province, School of Physics and Materials Engineering, Hefei Normal University, Hefei 230601, People's Republic of China

*E-mail: mayuan@hfnu.edu.cn; zfzi@issp.ac.cn

Received: 6 December 2021 / Accepted: 11 January 2022 / Published: 2 February 2022

Lithium metal anode is one of the most important potential materials in lithium-ion battery system because of its high energy density. In this paper, the problems of lithium metal anode are reviewed. Then, the application of 3D collector on lithium metal anode is summarized. 3D conductive collector is an effective way to solve this problem. The uniform deposition of lithium metal is realized by using high specific surface and low current density. The lipophilic performance of three-dimensional conducting collector was optimized by doping and lithium melting techniques. Finally, the research progress of gradient 3D graphene is investigated. Based on the literature survey, we believe that gradient 3D graphene is expected to be applied to lithium metal anode.

Keywords: Lithium metal anode; Gradient 3D graphene; 3D collector; Lithium dendrite

\section{$\underline{\text { FULL TEXT }}$}

(C) 2022 The Authors. Published by ESG (www.electrochemsci.org). This article is an open access article distributed under the terms and conditions of the Creative Commons Attribution license (http://creativecommons.org/licenses/by/4.0/). 